\title{
Information, Complexity and Generative Replication
}

\author{
Geoffrey M. Hodgson and Thorbjørn Knudsen
}

11 April 2007

Published in Biology and Philosophy

The Business School, University of Hertfordshire, De Havilland Campus, Hatfield, Hertfordshire AL10 9AB, UK* g.m.hodgson@herts.ac.uk* and

Department of Marketing and Management, University of Southern Denmark, Odense Campus, 5230 Odense M, Denmark

* Addresses for correspondence:

KEY WORDS: replication; replicators; information; complexity; automata

JEL classification: B52

\begin{abstract}
The established definition of replication in terms of the conditions of causality, similarity and information transfer is very broad. We draw inspiration from the literature on self-reproducing automata to strengthen the notion of information transfer in replication processes. To the triple conditions of causality, similarity and information transfer, we add a fourth condition that defines a "generative replicator" as a conditional generative mechanism, which can turn input signals from an environment into developmental instructions. Generative replication must have the potential to enhance complexity, which in turn requires that developmental instructions are part of the information that is transmitted in replication. Demonstrating the usefulness of the generative replicator concept in the social domain, we identify social generative replicators that satisfy all of the four proposed conditions.
\end{abstract}




\title{
Information, Complexity and Generative Replication
}

\author{
Geoffrey M. Hodgson and Thorbjørn Knudsen
}

\section{Introduction}

Considerable recent progress has been made in refining the abstract definitions of the core Darwinian concepts of variation, inheritance and selection. ${ }^{1}$ This work is guided by the insight that Darwinian principles do not apply to biological organisms alone, but to the evolution of a broad class of phenomena, including technologies and social institutions. ${ }^{2}$ If Darwinian principles have a general application to complex systems involving populations of varied and causally interacting entities, then we are obliged to specify these principles clearly in abstract terms, so they can serve as constructive principles for theory development across specific empirical domains.

This essay focuses on inheritance and replication. Since it was coined by Richard Dawkins (1976), the replicator concept has been clarified (Hull, 1988; Sterelny et al., 1996; GodfreySmith, 2000a; Sperber, 2000; Aunger, 2002; Nanay, 2002). However, efforts in this direction are complicated by the famous "evo-devo" dispute concerning the relative roles of development, selection and genotypic transmission (Baguñà and Garcia-Fernàndez, 2003; Gilbert et al., 1996; Stadler et al., 2001; Wimsatt, 1999). Recent formulations of "developmental systems theory" try to generalize Darwinian principles in very broad terms that sideline or exclude the replicator (Griesemer, 1994, 1999; Griffiths and Gray, 1994, 1997; Wimsatt, 1999). ${ }^{3}$

Theorists emphasizing the developmental side of the evolutionary process point out that inherited information itself cannot provide a complete description of the emerging phenotype and consequently development depends crucially on the particular context. Heredity and development interact in a way that cannot give overwhelming priority to the genotype. James Griesemer (1994) thus roots the concept of inheritance in whole developmental lineages, rather than in genotype-to-genotype transmission.

Paul Griffiths and Russell Gray (1997, p. 473) explain that developmental systems theory "takes to its logical conclusion the slow unraveling of the idea that genes are the sole evolutionary replicators." In particular, some biologists have "drawn attention to the large class of structures which are inherited parallel with the genes and play an essential role in development at the cellular level. ... These intra cellular elements of the developmental matrix are essential for the replication of DNA and are not themselves constructed on the basis of DNA sequences." As Eva Jablonka and Eörs Szathmáry (1995) argue, these parallel structures constitute an additional "epigenetic inheritance system". Hence developmental

\footnotetext{
1 The authors are very grateful to Marion Blute, David Hull, Pavel Luksha, Peter Richerson, Kim Sterelny, Viktor Vanberg, anonymous referees and several others for comments and discussions.

2 See Dawkins (1983), Hull (1988), Dennett (1995), Hodgson (2004, 2005) and Hodgson and Knudsen (2006a). ${ }^{3}$ Wimsatt (1999) rejects the replicator-interactor distinction but retains the genotype-phenotype concepts. This is
inconsistent if we accept the former distinction as a generalization of the latter (Brandon, 1996).
} 
systems theory shifts the focus onto the entire "developmental system" rather than the replicator and its "vehicle" (Dawkins, 1976) or "interactor" (Hull, 1988). While issues surrounding development are important, they do not, in our view, overturn the core Darwinian principles of variation, selection and inheritance.

Can sociocultural evolution be explained in terms of generalized Darwinian principles? Darwin $(1859,1871)$ himself considered the evolution of language and ethics. Other social scientists developed the idea more than a century ago (Bagehot, 1872; Ritchie, 1896; Veblen, 1899; Keller, 1915). The idea was revived thanks to Donald T. Campbell (1965) and others. Robert Boyd and Peter Richerson (1985), and William Durham (1991) have considered culture as an inheritance mechanism. However, this recent literature largely bypasses the question of the detailed mechanisms of cultural replication or transmission (Wimsatt, 1999). There is some ambivalence whether "memes" are genotypes or phenotypes, replicators or interactors (Blackmore, 1999).

Our aim is to explore in more detail the social and psychological mechanisms of sociocultural evolution, and where possible place these in a generalized Darwinian framework. Once we examine the transmission mechanisms involved, then we are presented with multiple possible substrates, from brain patterns to symbols and artefacts. Nothing in social culture remotely corresponds to the DNA code. As Stephen Jay Gould (1996) and William Wimsatt (1999) elaborate, there are further radical dissimilarities. For example, in the sociocultural domain there are complex patterns of inheritance and few clear boundaries between species. For Gould these are reasons to reject the idea of communalities between biological and sociocultural evolution. For Wimsatt they mean that evolutionary concepts have to be pushed to a high level of generality, after abandoning the distinction between replicators and interactors.

In contrast, we propose that the distinction between replicator and interactor can be retained in the sociocultural domain. Part of our rationale for this devolves from the understanding that Darwinian evolution is essentially about interacting and replicating populations of (varied and developing) entities rather than singular "self organizing" or "developing" systems. Once this focus on populations is established, then we need to understand the sources of both similarity and variation between elements, and to place this in the context of both the development of individual entities and the evolution of entire populations. We regard the replicator and interactor concepts as essential to this task. As Robert Brandon (1996, p. 125) argues, the distinction between replicators and interactors "is best seen as a generalization of the traditional genotype-phenotype distinction". Without the replicator and interactor, the distinction between genotype and phenotype similarly dissolves.

Although the possibility of multiple levels of selection has long been mooted by biologists and is well established in the philosophy of biology (Brandon, 1996; Keller, 1999; Michod, 1999; Sober and Wilson, 1998), it is sometimes sidestepped in discussions of replicators and interactors. It is especially pertinent in this context because what might emerge as an interactor at one level might conceivably act as a replicator at another (higher) level. For instance, some prominent accounts view human individuals as biological interactors and their genotypes as biological replicators. The admission of an additional and cultural level of transmission leads Boyd and Richerson (1985) and Durham (1991) to treat individual ideas or preferences as copied or transmitted in that "higher" domain. It is only one further step to consider social groups, structures or institutions as plausible interactors (Nelson and Winter, 1982; Henrich, 2004; Hodgson and Knudsen, 2004b). If this is a valid move, then the (human individual) interactors at the biological level become the repositories of replicator-like 
features (dispositions or preferences) at the sociocultural level. The question of multiple levels of selection has to be moved higher up the agenda.

Failure to do this might exacerbate the problems identified by Kim Sterelny et al. (1996); they argue that developmental systems theory lacks adequate definition of the boundaries of the units in the evolving population. We propose that some such boundaries must exist in an evolutionary system that is capable of retaining relatively successful adaptations and generating increasing complexity.

A second feature of our strategy is to make a link with the literature in mathematics and computer science on self-reproducing automata (Neumann, 1966; Sipper, 1998; Freitas and Merkle, 2004). John von Neumann's theory of self-reproducing automata has had little impact on the discourse on replication in the philosophy of biology. Yet it provides theoretical illumination and offers an additional context in which any generalized Darwinian theory must fit.

The remainder of this paper is organized as follows. In section two we scrutinize the widely used definition of replication in terms of the triple conditions of causality, similarity and information transfer. Section three draws from the literature on self-reproducing automata to strengthen the notion of information transfer in replication processes. Essentially, generative replication requires that developmental instructions are part of the information that is transmitted in replication.

Section four establishes the concept of generative replication. In addition to the triple conditions of causality, similarity and information transfer, we adopt a new condition that defines a generative replicator as a conditional generative mechanism, a material entity that embodies mechanisms that can turn input signals from an environment into developmental instructions. By this definition, genes but not prions are generative replicators. Section five examines the conjecture that generative replication has the potential to enhance complexity. Section six demonstrates the usefulness of the concept of generative replication in the social domain by identifying habits as social replicators that satisfy all four conditions. Section seven concludes the paper.

\section{Replicators and Replication}

Dawkins (1976) described replicators as having longevity, fecundity and fidelity. Dawkins (1982, 2004) also defined replication as involving genotypic copying fidelity sufficient to limit copying error and to pass on to successive generations the genotypic errors and mutations that actually occur. He identified both genes and "memes" as replicators, but the term "meme" was so broad that it encompasses ideas, practices, writings, paintings, artefacts, pop tunes and social institutions. There is ongoing controversy on what a meme is, and little progress in identifying the equivalent to the "genetics" of memetic replication. David Hull (1988, p. 408) defines a replicator as "an entity that passes on its structure largely intact in successive replications." A key question here is what structures are significant and why.

Sterelny et al. (1996, p. 395) offer a more elaborate definition of a replicator. They propose that if $\mathrm{B}$ is a copy of $\mathrm{A}$, and $\mathrm{B}$ is produced through a process of replication, then "A plays a causal role in the production of $\mathrm{B}$ " and "B carries information about $\mathrm{A}$ in virtue of being relevantly similar to A. This similarity is often functional: B has the same, or similar, functional capacities to A". Their definition emphasizes the three key points of causal 
implication, similarity and information transfer, which are central to most subsequent definitions.

These three elements, taken together, admit a very wide class of entities. Against Dawkins and others, Sterelny et al. argue that non-organisms such as bird nests and animal burrows qualify as replicators. But the causal role that one nest or burrow plays in the production of another is highly limited. If nests and burrows are to be admitted as replicators, then their causality condition has to be interpreted in very weak terms. The causal link is merely that each replicator is a practice model for its successors. Arguably the causality condition has to be coupled with other stipulations that make it less loose and more meaningful. Our definition below of "generative replicators" excludes nests, burrows, and many human artefacts.

In another development of the replicator concept, Peter Godfrey-Smith (2000a, p. 405) emphasizes that replication involves "two main elements, a resemblance between copy and copied, and some suitable causal relation linking the copy to the copied." Like Dan Sperber (2000), he is notably sceptical of versions of cultural evolution based on the meme.

Dan Sperber (2000, p. 169) argues that replication involves three elements of causation, similarity, and information transfer. ${ }^{4} \mathrm{He}$ refines the information transfer condition as follows: "The process that generates B must obtain the information that makes B similar to A from A. ... B must inherit from A the properties that make it relevantly similar to A." He argues that many cases of so-called memetic replication are not true replication, principally because his information transfer condition is violated.

A problem is that the three conditions of causality, similarity and information transfer cover a broad class of copying processes. They would also apply to technologies such as photocopying, and the copying of data on magnetic media or on compact disks. The similarity condition pertains. The causality condition is also satisfied because the original is causally implicated in the production of the copy, in the weak sense that without the original the copy could not exist. For Sperber (2000) the "information transfer" condition is also satisfied because the "information" in the copy originates from the original.

Although these criteria cover a broad range of copying processes, Sperber points to exceptions, such as the example of contagious laughter spreading through a group. Although one person's laughter may trigger the laughter of another, the laughter itself is "not copied" because the second person does not imitate the laughter of the first. Instead "there is a biological disposition to laughter that gets activated and fine tuned through encounters with the laughter of others. ... The motor program for laughing was already fully present in him, and what the laughter of others does is just activate it" (Sperber, 2000, p. 168). He thus claims that his information transfer condition is unsatisfied in this case.

If instead a second sound recorder were switched on to record the sounds of a first recorder, then for Sperber this would be a case of true replication satisfying all three of his conditions. Similar remarks seem to apply to the photocopying of documents. However, while these examples are different from the contagious spread of laughter, the difference seems mainly of degree. The triggering of patterns of laughter may involve a signal that is much simpler than the detailed information copied by sound recorder or photocopier, but the difference lies in the

\footnotetext{
4 Aunger (2002) refines Sperber's (2000) definition by adding a fourth condition of "duplication", meaning that replication of one entity gives rise to two or more. We are unconvinced that Aunger's fourth condition of "duplication" is necessary or useful. The critical issue in understanding the process of replication is the nature and function of the structure that is passed on, including any information that it might hold.
} 
amount and the kind of information transferred. In no case is the transferred information sufficient itself to produce the copy. In none of these cases is the copying mechanism itself copied.

What if the intensity and style of the initial laughter triggers mirth of a similar intensity and style in others? Then the initial laughter would be carrying information that was critical in forming the character of the copied laughter. Sperber himself admits the possibility of laughter being "fine tuned" through encounters with others. In which case the initial laughter becomes more than a mere trigger: it carries significant information that is copied.

Furthermore, Sperber's condition that "B must inherit from A the properties that make it relevantly similar to A" can be interpreted in terms that exclude not only his example of laughter, but also the examples of copying with sound recorders or photocopiers. It all depends what we mean by "properties". In his discussion of laughter, Sperber focuses on the capacity to produce the behaviour. The disposition to laugh is not replicated, but neither is the capacity to make copies with a sound recorder or photocopier. These machines already have the capacity to make copies. Hence, if these "properties" include the capacity to produce a copy, as well as the information in the copy, then none of these examples satisfy his information transfer condition, because some of these key properties are not inherited in the copying process.

Although Sperber's information transfer condition takes us in the right direction, it is insufficiently precise. It depends crucially on what is meant by "information" and what properties have to be inherited to constitute true replication. A critical issue pertains to the replication of a copying mechanism, or the absence of such processes. Relevant insights appear in our discussion of self-reproducing automata in the next section.

\section{Inspiration from Self-Reproducing Automata}

John von Neumann (1966) considered conditions under which automata would be capable of producing copies of themselves, and capable of generating novelty and additional complexity. He did not propose that evolution always increases complexity, but he was interested in the conditions under which further complexity could be generated. Von Neumann (1966, p. 80) distinguished between the copy of entities that synthesized to produce higher degrees of complexity, and the copying of entities that reduced overall complexity:

There is thus this completely decisive property of complexity, that there exists a critical size below which the process of synthesis is degenerative, but above which the phenomenon of synthesis, if properly arranged, can become explosive, in other words, where synthesis of automata can proceed in such a manner that each automaton will produce other automata which are more complex and of higher potentialities than itself.

Following von Neumann, we propose that generative replication should be confined to cases with the potential to increase complexity. This would exclude copying with sound recorders or photocopiers, or of nests and burrows. Such copies have no more potential to enhance complexity than their predecessors. For increasing potential complexity, copies must eventually be capable of producing novel additional components, or performing novel operations in response to new environmental conditions and input signals.

Von Neumann (1966) examined in detail the properties that a self-reproducing automaton must possess to make it capable of producing other automata. These properties include instructions that describe the structure and processes of an automaton, a copying unit that is 
capable of reading and copying instructions into a new automaton and translating them as directions for a production unit, a production unit that builds a new automaton, and some mechanism of coordination between these elements. His research has influenced robotics and computer science (Sipper, 1998; Luksha, 2003).

However, biological replicators lack the abstract properties of self-reproducing automata outlined by von Neumann. In particular, as developmental systems theorists emphasize, genes themselves do not carry enough "information" to describe or generate a new organism. The development of the organism depends additionally not only on environmental stimuli, but also on cellular structures inherited alongside the genes. Consequently, neither genes nor organisms constitute a complete "production unit" for new organisms.

The theory of self-reproducing automata points to the potential to enhance complexity. It also sees the developmental instructions as part of the information that is transmitted on replication. These two inspirations lead us to the concept of generative replication. A generative replicator is a material entity that is responsive to environmental stimuli or signals. There is at least one signal that can cause a non-degenerative response from the replicator. Such a response consists of further instructions or signals to the interactor, which guide its development. It is non-degenerative in the sense that it leads to outcomes that are conducive to the survival of the replicator and the information it carries.

For example, the DNA "code" determines the constitution of the protein molecules in the organism. It instructs the processes of cell-formation by governing the production of amino acids and proteins, subject to the circumstances and external conditions involved.

\section{The Generative Replicator Defined}

We acknowledge Dawkins's point about copying fidelity and passing on mutations, but it is insufficient for our project. Our concern here is to define a special class of replicators that have the potential to increase complexity, rather than to define the broader boundaries of replication itself. Furthermore, Dawkins (2004, p. 391) associates copying fidelity with mutations or errors in genotypic information and by contrast requires that most 'environmentally acquired changes' are not passed on. But this important distinction begs the definition of a genotype or replicator.

On the basis of our discussion of self-reproducing automata, we adopt an additional fourth proposition in the definition of a generative replicator:

4. Conditional generative mechanisms: Generative replicators are material entities that embody construction mechanisms (or "programs") that can be energized by input signals, containing information about a particular environment. These mechanisms generate further instructions from the generative replicator to the interactor, to guide its development. (External influences that produce outcomes generally unfavourable to the survival of the replicator or interactor are not described as input signals but as destructive forces.)

This first proposition adds to the established three definitional features for a replicator, namely causal implication, similarity and information transfer. We refine these as follows:

1. Causal implication: The source must be causally involved in the production of the copy, at least in the sense that without the source the particular copy would not be created. 
2. Similarity: The replicated copy must also possess the capacity to replicate. The conditional generative mechanisms in the copy must be similar to those in the source. Errors or mutations in these mechanisms must also be copied with some degree of fidelity.

3. Information transfer: The process that generates the copy must obtain the conditional generative mechanisms that make the copy similar to its source from that same source.

Note how the causality condition is clarified. The enhanced similarity condition requires that similarity must apply to the conditional generative mechanisms and takes on board Dawkins's (2004) stipulation. A related refinement appears in the information transfer condition. These conditions preserve the spirit of Hull's (1988, p. 408) definition of a replicator as "an entity that passes on its structure". An entity that satisfies all these four conditions is described as a generative replicator.

One of the problems in defining the replicator concept is understanding what exactly "information" means. Our specification helps to fill this gap. The concept of information here does not necessarily carry interpretations or meanings in the same way as the information communicated by humans. It is information in a cruder sense of a code or signal, as stored and manipulated by computers and present in the DNA. For Claude Shannon and Warren Weaver (1949) a message has "information content" when its receipt causes some action. For us, the "information" involved consists of signals with the potential to trigger generative mechanisms that guide the production of further replicators or the development of interactors.

Hence "information" in our definition is not ideas, which necessarily involve meanings and interpretations. Our added condition insists on the materiality of the replicator, hence ideas as such are not replicators. More appropriately, ideas may be regarded as emergent expressions of mental habits or dispositions. By contrast, habits qualify as generative replicators, as shown below.

Our concept of "conditional generative mechanism" is close to what Ernst Mayr (1974, 1988) describes as a "program". Mayr (1988, p. 48) regards a program as something embodied in a material substrate that gives rise to goal-driven (or "telenomic") behaviour, and hence is "consistent with a causal explanation".

Nests, burrows and photocopies are not generative replicators. None of these entities is capable of receiving and emitting signals that lead to the development of the interactor. However, given that their survival is dependent on specific environmental conditions, then changes in those conditions can lead to changes in their state. They can be destroyed by (say) water or fire. To exclude such destructive influences, the sentence in parenthesis in condition (4) above establishes that destructive or degenerative environmental influences do not count as signals. Having made this exclusion, nests, burrows and photocopies have no conditional mechanisms to guide the development of the interactor.

Dawkins rejects the selfish nest and similar examples on the grounds that they fail to meet the similarity condition - small copying errors in nest and burrow production are not preserved and transmitted to a third generation. Such preservation is essential for cumulative evolution via natural selection, and cumulative evolution is essential for the evolution of complex adapted structure. For Dawkins, replication in conjunction with the right kind of fidelity is the necessary foundation of complexity.

Our analysis complements that of Dawkins, who characterizes the output conditions of inheritance mechanisms if complex systems are to evolve. In contrast, we consider the 
mechanisms that are required to generate complex outputs. We exclude nests and burrows because they do not contain a mechanism that can receive and emit signals that lead to the further development of the interactor. Hence they cannot compress, transmit and express information required in building structures of increasing complexity; they exhibit limited heredity in the sense of Maynard Smith and Szathmary (1995). We identify generative replication as a necessary condition for the evolution of structures which are potentially unbounded in complexity. The emergence of unlimited heredity requires generative replication.

Briefly consider some further examples. Widely regarded as replicators, prion proteins are associated with transmissible spongiform encephalopathies (such as BSE). They involve the accumulation of an abnormally folded variant of the normal prion protein, which spreads by direct contact when the normal form also becomes misfolded and thus converted to an abnormal and equally infectious form (Prusiner, 1998). The replication of the abnormal form first grows exponentially and then dies out when the pool of normal prion molecules is exhausted. Prion replication does not contain signal-responsive construction mechanisms that hold information about a particular environment. At most, it is a simple form of replication where no conditional generative mechanisms are passed on. Hence prions are not generative replicators.

Biological viruses are infectious organisms with a nucleic acid genome (DNA or RNA). They spread by genome replication rather than direct contact. The genome of a virus involves a signal-responsive construction mechanism. A newborn genome codes for the protein coat (capsid) within which it is then housed. Thus, viruses are interactors (the protein coat) that house a replicator (the genome). Biological viruses hold information about special environments and acquire adaptive solutions to particular problems (such as immune response). Their hosts are not their interactors, and even if biological viruses are destructive for their hosts, they qualify as generative replicators.

Computer viruses also contain signal-responsive construction mechanisms. Computer viruses also hold information about special environments (e.g. particular types of operating systems) and some even acquire adaptive solutions to particular problems (e.g. to avoid detection). Computer viruses are replicators, and their interactors are arguably the computer programs they infect. ${ }^{5}$ This contrasts with biological viruses, whose hosts do not constitute their interactors. Consequently, because computer viruses are generally destructive for the programs they infect, they are not generative replicators. ${ }^{6}$

\section{Generative Replication and Complexity}

For two centuries it has been debated whether evolution generally gives rise to increased complexity (Adami, 2002; Adami et al., 2000; Gould, 1977; Saunders and Ho, 1976, 1981). Some endorse the proposition, some suggest the evidence is inconclusive, and others reject the idea.

\footnotetext{
5 Note Hull's (1988, p. 408) definition of an interactor as "an entity that directly interacts as a cohesive whole with its environment in such a way that this interaction causes replication to be differential." See Hodgson and Knudsen (2004b) for a refinement of this definition. 6 Even so-called benign computer viruses can be mildly destructive for the computer systems they infect, by
taking up computer memory, causing erratic performance or triggering system crashes.
} 
The disagreement also concerns what definitions and measures of complexity to use when such claims are assessed (Adami, 2002; Adami et al., 2000). Christoph Adami's (2002) useful review of definitions and measures of complexity concluded that many have drawbacks. Consistent with mathematical information theory, Adami upholds that the essence of complexity for an evolving entity is the amount of information that it stores about the environment in which it evolves. Adami (2002, p. 1087) writes:

The physical complexity of a sequence refers to the amount of information that is stored in that sequence about a particular environment. For a genome, this environment is the one in which it replicates and in which its host lives, a concept roughly equivalent to what we call a niche. Information ... is always about something. Consequently, a sequence may embody information about one environment (niche) while being essentially random with respect to another. This makes the measure relative, or conditional on the environment, and it is precisely this feature that brings a number of important observations that are incompatible with a universal increase in complexity in line with a law of increasing physical complexity.

This definition conceptualizes complexity as information relative, and conditional on the environment in which the entity evolves. Information is obtained from input signals that are actually recorded in a sequence stored in a replicator (genome), which in turn requires a distinction between replicators and interactors. These aspects of complexity are captured by our proposition that a conditional generative mechanism is a defining feature of a generative replicator in the sense that it is a material entity embodying mechanisms that can be energized by input signals.

We adopt here the conventional concept of information from mathematical information theory (Shannon and Weaver, 1949). Complexity corresponds to the amount of information that a replicator stores about a particular environment. The stored information relates the state of a replicator sequence to the actual state of the environment. A replicator sequence whose state perfectly corresponds to the state of the environment has maximal information about the environment in question.

Complexity is measured as (neg)entropy. If the entropy of a replicator population increases, then it contains less information about an environment. As explained below, we measure complexity as the difference between the theoretical maximum amount of information about an environment and the actual entropy (disorder) present in the relevant replicator population. As this difference increases, the replicator population exhibits less disorder and more complexity and contains more useful information about the environment. By contrast, if there is a diminishing difference between the maximum amount of information and actual entropy (of replicators), then a replicator population looses track of the environment and exhibits less physical complexity.

Before defining complexity formally, we briefly consider how our appeal to information relates to the recent controversy about information concepts in biology (Godfrey-Smith, 2000b; Griffiths, 2001; Maynard Smith, 2000a, 2000b; Sarkar, 2000; Sterelny, 2000). Griffiths (2001) suggests a distinction between causal and intentional information. Our use of the information concept belongs squarely to the causal category. However, some find that it does not go far enough to characterize the nature and function of the gene, because causal information concepts apply equally to genetic and environmental factors. Thus Maynard Smith appealed to a stronger symbolic or intentional concept of information to characterize the way genes embody evolved properties that are expressed in particular phenotypical features. 
We do not intend here to contribute to this controversy and our argument does not depend on its outcome. Rather, we use an information concept that is widely accepted and commonly used across a number of scientific disciplines. This implies that information and complexity, as defined here, applies equally to genetic and non-genetic factors; accordingly the "parity thesis" from mathematical information theory applies.

We do not suggest that genes or replicators are special because they contain a particular kind of information. Instead we suggest that replicators differ on whether or not they contain a generative mechanism. Generative replicators have the capacity to increase complexity. Like other generative replicators, genes have this special quality.

Our more detailed definition of the complexity of a replicator population largely follows Adami's (2002) exposition. Consider a specific environment $E$ and the features required of a notional generative replicator to maximize interactor fitness in this environment, captured by a binary string of length $L$ (the number of binary bits used to describe the generative replicator). ${ }^{7}$ The theoretical entropy value of these notional fitness maximizers, $H_{\max }$, is simply the sequence length $L$. Population level complexity is the difference between $H_{\max }$ and the entropy of the actual population of generative replicators operating within this environment, also described in terms of binary strings of length $L$.

To determine the entropy of the actual population of generative replicators, each bit is addressed in turn. For the population as a whole, the actual frequency of the (binary) fitnessmaximizing value at locus $i$ is $p_{i}$ (where $0 \leq p_{i} \leq 1$ ). The entropy of a population of generative replicators $X$ is denoted by $H(X)$. The measure of physical complexity $C$ of a population of replicating entities is the information that the generative replicator sequences $X$ contain about the environment $E$ :

$$
C=H_{\max }-H(X)=L+\Sigma p_{i} \log p_{i}
$$

Our definition of generative replication is based in part on the conjecture that the capacity to increase complexity depends critically on the existence and replication of a conditional generative mechanism. We illustrate this by considering three types of error. ${ }^{8}$ Namely, there is reading error (concerning input signals), developmental error (concerning the development of interactor traits from the generative replicator) and copying error (from a generative replicator to its offspring). A weaker surmise is that copying errors among a population of generative replicators are generally more destructive to complexity than reading and developmental errors. A stronger proposition is that the capacity to increase complexity depends critically and especially on generative replication with low copying errors. We offer some arguments below in favour of the weaker surmise, and we refer to some simulation work that we have done that illustrates the stronger proposition.

Consider a process of repeated replication with input signals, the development of interactors and the copying of generative replicators, as in figure 1 . We assume that there is no alteration of stored information in any individual generative replicator, implying no Lamarckian inheritance. In a fixed environment, these generative replicators develop traits clustered around a peak in a fixed fitness landscape. (Changing environments and variable or "dancing"

\footnotetext{
${ }^{7}$ We use a binary string as a useful simplification for expositional reasons.

8 Prior research on self-replicating automata (Molofsky, 1994; Wolfram, 1984, 2002) has typically excluded such errors.
} 
fitness landscapes are excluded for simplicity, not because they would undermine our argument.)

Both reading errors and developmental errors have a similar type of effect: they cause the population to disperse from its original position in the fitness landscape. However, if copying errors are zero, and the probabilities of reading and developmental errors remain constant, then the trend of overall dispersion will not increase through time as repeated replication occurs. The effects of reading and developmental errors are not cumulative. Information content is preserved through the faithful copying of the generative replicator.

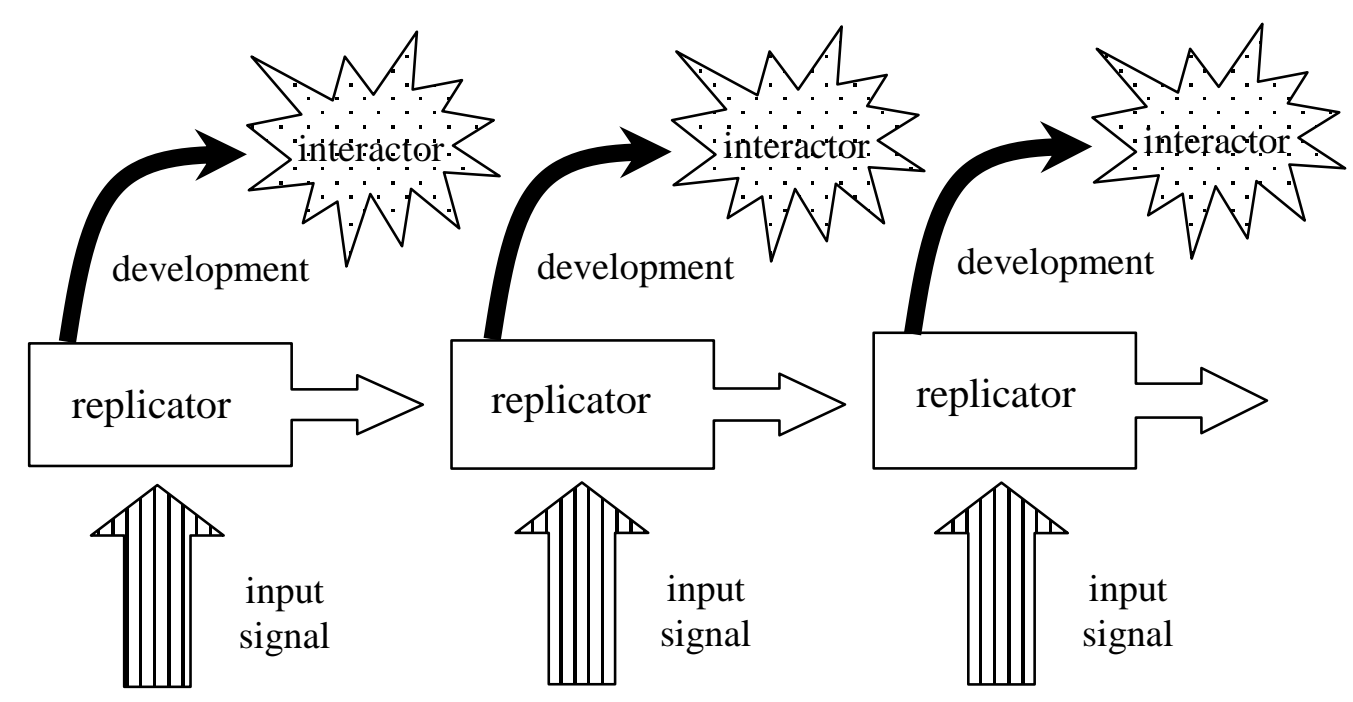

Figure 1: Generative replication with Input Signals and Interactors

Re-start the process but assume that the probability of a copying error is positive. The population of generative replicators is again clustered around a peak in a fixed fitness landscape. If a generative replicator is close to the fitness peak, then copying error is likely to move it away from that peak. There will be no reliable mechanism to tie it to its previous position. A process of drift will occur and the overall dispersion in the population will increase. Hence the effects of copying error are cumulative. The entropy $H(X)$ of the replicator population will increase and overall complexity will decline as a result. ${ }^{9}$

This demonstration that copying error is especially destructive to complexity illustrates the special importance of two of our features of a generative replicator, concerning the existence and relatively faithful copying of a conditional generative mechanism. It is this mechanism that has the potential to generate structures which are potentially unbounded in complexity (a critical condition in Dawkins's analysis). We acknowledge that the above argument depends

9 Note that, if it did exist, "Lamarckian" inheritance could have similarly negative cumulative effect. Lamarckism upholds that the acquired characteristics of the interactor may affect the replicator, leading to the inheritance of these acquired characteristics. In biology, Lamarckian inheritance is widely ruled out because of the Weismann barrier. Although social evolution is widely described as Lamarckian, this idea is contested (Hodgson and Knudsen, 2006b). 
on restrictive assumptions. Nevertheless it underlines the importance of copying fidelity and the cumulatively destructive effect on complexity of copying error.

We admit that destructive forces, which reduce the chances of survival of some generative replicators, can undermine complexity in a population. The occurrence of disasters or extinctions may reverse any trend towards greater complexity. Our argument is based on the potential to increase complexity, and not necessarily its empirical manifestation through time. Furthermore, there are additional sources of complexity other than replication.

To substantiate the proposition that the capacity to increase complexity depends critically on generative replication with low copying errors, we conducted a number of simulation experiments (Hodgson and Knudsen, unpublished). We used the classical one dimensional linear automaton, specified by binary values in a line of cells. At each time step, there is replication of a rule (an information sequence comprising the replicator) and a subsequent process of development where the generative replicator is energized by input signals. The outcome of the development process is a new individual entity whose trait value depends on the way the generative replicator reads and codes input signals.

We found that even low levels of copying error can thwart the enhancement of complexity, and this is undermined still further as the level of copying error is increased. However, selection pressure can diminish the destructive effects of copying error. Uppermost, we showed that only if replicators comply adequately with all four of our definitional conditions, then complexity will increase towards some maximal level. Adami et al. (2002) reach a result that is consistent with this conclusion. ${ }^{10}$

Our argument that generative replication furthers complexity has striking similarities to that of Ridley (2000). Ridley's premise is similar to ours that deleterious mutation must be kept within bounds if evolution is to occur. If the average rate of deleterious mutations is too high, then deleterious mutations will cumulate and quickly swamp the descendant populations.

Like us, Ridley sees a tension between the evolution of complexity and the encoding of information. The evolution of complexity necessarily involves encoding more information about an environment. This requires a larger replicator sequence ( $L$ in equation 1$)$. However, a longer replicator sequence will also more likely hit the critical threshold of one deleterious mutation per copy. The evolution of complexity requires that copying error is kept below a critical threshold.

Ridley focuses on mechanisms that minimize copying error. He convincingly explains how meiosis diminishes the probability that deleterious mutations will swamp a population. By independent assortment and recombination, deleterious mutations are spread thin, so selection maintains a basis for ridding the population of such mutations.

Ridley's argument translates into the proposition that the maximal level of complexity is determined by the length $L$ (equation 1 ) of a replicator sequence and that the length $L$ is determined by the level of copying error. The evolution of mechanisms that decrease copying errors in generative replication will therefore enable the evolution of complexity. Known examples include a storage medium that does not degrade its content (e.g. DNA over RNA), dilution of deleterious mutations through randomized combinatorics (meiosis), error checking and DNA repair, as well as sexual mating. When such mechanisms evolve, the limit of complexity to be achieved in a population of generative replicators will increase.

\footnotetext{
10 We used sequences of length 100, but additional simulations show that our results are robust for much longer sequences.
} 


\section{Habits as Social Generative Replicators}

Attempts to extend Darwinian principles to social or cultural evolution are as old as Darwinism itself. However, detailed discussions of the units and mechanisms of social or cultural replication are rare. It is important to make progress on this front if Darwinian theories of social or cultural replication are to develop further.

We have proposed that habits are elemental social replicators in the social world (Hodgson, 2003; Hodgson and Knudsen, 2004a, 2004b). A habit is a disposition to engage in previously adopted or acquired behaviour; triggered by an appropriate stimulus. Habits are formed through repetition of behaviour or thought. They are influenced by prior activity and are the basis of both reflective and nonreflective behaviour. Crucially, we may have habits that lie unused for a long time. Habits are submerged repertoires of potential behaviour that can be triggered by an appropriate stimulus or context.

Habits sometimes give rise to observable behaviour and are sometimes confined to unobservable thoughts. Although all habits involve thoughts or mental activity, for simplicity we refer to the former as "habits of behaviour" and the latter as "habits of thought". Unlike DNA or computer viruses, habits of behaviour do not directly make copies of themselves. Instead they replicate indirectly, by means of their behavioural expressions. They can impel behaviour that is consciously or unconsciously followed by others, as a result of incentive or imitation. Eventually, the copied behaviour becomes rooted in the habits of the follower, thus transmitting from individual to individual an imperfect copy of each habit.

The replication of observable habits of behaviour satisfies all the four criteria for generative replication. Both the original habit and its copy embody a conditional generative mechanism. The acquired habit is both energized conditionally on the receipt of environmental signals and plays a constructive role in the development of the interactor, i.e. the individual with the habit. Furthermore, the habit of behaviour in one person causes behaviour that is copied, at least in the sense that the copy depends on the source, and leads to similar habits being acquired. The acquired habit of behaviour is similar to the first with respect to the behaviour it might promote under specific conditions. Tacit or other information is transferred in the process. ${ }^{11}$

Habit-forming behaviour can be moulded by incentives or constraints. In many cases, such as language or some traffic conventions, we can have powerful incentives to behave like others. In doing so, we too build up habits associated with these behaviours. The behaviours are reproduced and the habits giving rise to them are replicated.

Habit replication also often relies on imitation, which need not be fully conscious and may involve some "tacit learning" (Polanyi, 1967; Reber, 1993; Knudsen, 2002a). Imitation can result from an instinctive propensity, which has itself evolved for efficacious reasons among social creatures (Boyd and Richerson, 1985; Simon, 1990; Tomasello, 1999). If imitation is more than mimicry, then the rules and understandings associated with it also have to be transmitted.

By contrast, habits of thought are unobservable and cannot emerge through behavioural imitation. They consist of mental models that enable conscious deliberation and manipulation of situations. Actors adapt and use their mental models as guides as they proceed through a particular cultural context (Johnson-Laird, 1981). As habits, mental models are formed by

\footnotetext{
11 Similarity in neural connection design, even with similar genes and environment, is very unlikely (Edelman, 1989). Underlying neuronal configurations supporting similar habits are likely to differ between individuals.
} 
repetition of particular associations and patterns of thought. Under specific conditions, when agents experience common external constraints or regularities, they may develop similar mental models that will direct conscious deliberation towards a particular object in a particular class of situations. Different people that face similar environmental regularities will experience similar mental models that may be caused by different patterns of neuronal activity (Kurthen, 2001). Hence similarities in habits of thought and mental models can emerge when enduring similarities in external constraints or conventions exist.

Members of a species are similar regarding their physical construction, sensory organs, nervous system and brain function. These similarities provide common constraints that may promote some similarity in mental experiences of the external world; similarities in physical construction become causes as well as evolutionary consequences of similarities in experience. The possibility arises that two human beings will experience similar mental phenomena when confronted with the same external object (Edelman, 1989; Kubovy and Epstein, 2001).

Cultural circumstances and social institutions vary from society to society, but members of each society have to adapt to them. An evolutionary explanation of the replication of habits of thought or other mental phenomena requires and explanation of the evolution of the social institutions that provide common conventions or constraints. Common conventions or constraints are a necessary but insufficient requirement for the replication of habits of thought to take place.

However, if two agents in the same context independently produce similar mental models, then no replication has taken place because no mental model is causally implicated in the emergence of the other. Replication of mental models would require that one agent could somehow access the mental model of another. This raises the question of how crucial information in the replication process is communicated.

Language becomes paramount. In psychology and neuroscience, the acquisition of language initially establishes a triadic correspondences of mental models, objects and behaviours that become mental models common to the members of a society (Karmiloff and Karmiloff-Smith, 2001; Tomasello, 1999). The learning of a language involves the development of an elaborate correspondence between mental phenomena and properties of the physical and social world.

Through a shared language, a person can access the mental model of another. This transmission of mental models is improved by close interaction with error-correction. By gestures and questions, agents establish joint attention that increases the accuracy of transmission of mental models and establishes mutual understandings. Language is a vital link in this causal chain: the replication of habits of thought depends on language.

Habits of thought satisfy all four of our conditions for a generative replicator. They constitute conditional generative mechanisms that are essential to a generative replicator. They are energized conditionally on the receipt of external signals and play a role in the development of the individual. Given the existence of a shared common language as well as common extralinguistic points of reference, the habit of thought in one person causes a mental model that is transmitted and can lead to a similar habit of thought being acquired. The acquired habit of thought is similar to the first with respect to the mental model it might promote under specific conditions. Some kind of information regarding a perceived property of the world is transferred in the process. 
Genes themselves depend on the biochemical substrate of an organism. Similarly, habits cannot exist apart from the human organisms in which they reside. They are formed and stored in the individual human nervous system. But habits differ from genes in their mechanism of replication, and habits do not have anything like the potential durability and copying fidelity of the gene. In social evolution, additional mechanisms weed out or alter aberrant habits. Mechanisms of social conformity are particularly important (Henrich and Boyd, 2001).

Having established (observable and unobservable) habits as elemental generative replicators in the social world, we have the building blocks to understand other social generative replicators. These include customs and routines (in the technical sense of the term employed by evolutionary economists such as Nelson and Winter, 1982). These social generative replicators involve structured assemblies of habituated individuals. Behavioural cues by some trigger specific habits in others. Hence various individual habits sustain each other in an interlocking structure of reciprocating individual behaviours. But we have not got the space to go into the details here.

\section{Conclusions}

Inspired by the work of von Neumann on self-reproducing automata, we have established the concept of a materially-grounded conditional generative mechanism (or program) and argued that it plays a key role in the generative replication process. This mechanism is an essential part of the "information" that is stored in the generative replicator and copied through replication. It also informs and guides the development of the interactor.

Following von Neumann, we associate generative replication with the potential to increase complexity, given other vital factors, without assuming that evolution necessarily embodies such a trend. We are not saying that generative complexity always leads to greater complexity, or that complexity results from replication alone. We argue that copying error is generally more destructive to complexity than other forms of error, particularly in environmental interactions or individual development. With protein synthesis, the work of Lee et al. (2006) supports this conclusion by identifying a catastrophic loss of accuracy associated with accumulation of error.

The conditional generative mechanism appears in a four-clause definition of the generative replicator. Genes clearly qualify as generative replicators. Other suggestions, including bird nests, burrows, and photocopiers do not qualify because they lack a conditional generative mechanism, and their replication does not have the potential to increase complexity. Hence our definition has important discriminating powers and identifies an important subclass of replicators. However, we acknowledge that many processes in evolution do not rely on generative replication.

One might wish to go further and disregard all non-generative replicators as true replicators. We do not pursue this issue here. The degree of viable extension of the replicator concept, and the distinction between replication and contagion or diffusion, are issues that require further discussion (Sterelny et al., 1996; Szathmáry, 2000). Like Dawkins we emphasize genotypic copying fidelity, but we associate it with the special class of generative replication, rather than replication per se. Furthermore, our generative replicators are a subset of Dawkins's replicators, because our definition also requires material entities that embody developmental mechanisms (programs) that can be energized by input signals. Entities that replicate such developmental mechanisms can compress, transmit and express the information 
required in building structures of increasing complexity. Generative replication is therefore a necessary condition for heredity of structures which are potentially unbounded in complexity. Accordingly, the emergence of unlimited heredity requires generative replication.

Dawkins characterizes the conditions on the outputs of inheritance mechanisms for complexity to evolve, involving a potentially unlimited stock of distinct replicators in conjunction with the right kind of fidelity. In contrast, we consider the mechanisms that are required to generate one of those outputs, namely complexity.

Turning to sociocultural evolution, our refined definition has important implications. As widely acknowledged, the literature on memes suffers from vagueness and ambiguity. If memes are simply ideas, then our materiality condition rules them out as replicators. Instead, we regard ideas as emergent expressions of habits that in turn qualify as generative replicators. This overcomes the dualism and separation of the ideal and material worlds, by grounding ideas upon habits, as in pragmatist philosophy (Joas, 1993; Diggins, 1994; Putnam, 1995; Hodgson, 2004). We suggest that customs and (organizational) routines also qualify as generative replicators. Consideration of their mechanisms of replication would address the detailed equivalent of the "genetics" of social replicators that has been missing from discussions of memes.

We have underlined the importance of copying fidelity in information transmission from one generative replicator to another. Upon this the preservation and potential enhancement of complexity depends. A case can be made in the biological world that the Weismann barrier limiting any interference by the interactor with the information in the generative replicator has evolved to deal with this problem (Maynard Smith and Szathmáry, 1995). A big question is whether there is an equivalent Weismann barrier in the social domain. Genetic or culturally transmitted mechanisms of conformism may be important in sustaining such a barrier. This is among several research questions prompted by our definition of a generative replicator. 


\section{References}

Adami, Christoph (2002) 'What is complexity?', BioEssays, 24(12), pp. 1085-94.

Adami, Christoph, Ofria, Charles, and Collier, Travis C. (2000) 'Evolution of Biological Complexity’, Proceedings of the National Academy of Sciences, 97(9), pp. 4463-8.

Aunger, Robert (2002) The Electric Meme: A New Theory of How We Think (New York: Free Press).

Bagehot, Walter (1872) Physics and Politics, or, Thoughts on the Application of the Principles of 'Natural Selection' and 'Inheritance' to Political Society (London: Henry King).

Baguñà, Jaume and Garcia-Fernàndez, Jordi (2003) 'Evo-Devo: The Long and Winding Road', International Journal of Developmental Biology, 47, pp. 705-13.

Blackmore, Susan (1999) The Meme Machine (Oxford: Oxford University Press).

Boyd, Robert and Richerson, Peter J. (1985) Culture and the Evolutionary Process (Chicago: University of Chicago Press).

Brandon, Robert N. (1996) Concepts and Methods in Evolutionary Biology (Cambridge and New York: Cambridge University Press).

Campbell, Donald T. (1965) 'Variation, Selection and Retention in Sociocultural Evolution', in Barringer, H. R., Blanksten, G. I. and Mack, R. W. (eds) (1965) Social Change in Developing Areas: A Reinterpretation of Evolutionary Theory (Cambridge, MA: Schenkman), pp. 19-49. Reprinted in General Systems, 14, 1969, pp. 69-85.

Darwin, Charles R. (1859) On the Origin of Species by Means of Natural Selection, or the Preservation of Favoured Races in the Struggle for Life, $1^{\text {st }}$ edn. (London: Murray).

Darwin, Charles R. (1871) The Descent of Man, and Selection in Relation to Sex, $1^{\text {st }}$ edn., 2 vols (London: Murray and New York: Hill).

Dawkins, Richard (1976) The Selfish Gene (Oxford: Oxford University Press).

Dawkins, Richard (1982) The Extended Phenotype: The Gene as the Unit of Selection (Oxford: Oxford University Press).

Dawkins, Richard (1983) 'Universal Darwinism', in D. S. Bendall (ed.) (1983) Evolution from Molecules to Man (Cambridge: Cambridge University Press), pp. 403-25.

Dawkins, Richard (2004) 'Extended Phenotype - But Not Too Extended. A Reply to Laland, Turner and Jablonka', Biology and Philosophy, 19(3), June, pp. 377-96.

Dennett, Daniel C. (1995) Darwin's Dangerous Idea: Evolution and the Meanings of Life (London and New York: Allen Lane, and Simon and Schuster).

Diggins, John Patrick (1994) The Promise of Pragmatism: Modernism and the Crisis of Knowledge and Authority (Chicago: University of Chicago Press).

Durham, William H. (1991) Coevolution: Genes, Culture, and Human Diversity (Stanford: Stanford University Press).

Edelman, Gerald M. (1989) The Remembered Present: A Biological Theory of Consciousness (New York: Basic Books). 
Freitas, Robert A. and Merkle, Ralph C. (2004) Kinematic Self-Replicating Machines (Georgetown, TX: Landes Bioscience).

Gilbert, Scott F., Opitz, John M. and Raff, Rudolf A. (1996) 'Resynthesizing Evolutionary and Developmental Biology’, Developmental Biology, 173(2), February, pp. 357-372.

Godfrey-Smith, Peter (2000a) 'The Replicator in Retrospect', Biology and Philosophy, 15, pp. 403-23.

Godfrey-Smith, Peter (2000b) 'Information, Arbitrariness, and Selection: Comments on Maynard Smith’ Philosophy of Science, 67(2), pp. 202-207.

Gould, Stephen Jay (1977) Ontogeny and Phylogeny (Cambridge, MA: Harvard University Press).

Gould, Stephen Jay (1996) Life's Grandeur: The Spread of Excellence from Plato to Darwin (London: Cape).

Griesemer, James R. (1994) 'Tools for Talking: Human Nature, Weismannism and the Interpretation of Genetic Information', in Cranor, Carl (ed.) (1994) Are Genes Us? The Social Consequences of the New Genetics (New Brunswick, NJ: Rutgers University Press), pp. 69-88.

Griesemer, James R. (1999) 'Materials for the Study of Evolutionary Transition', Biology and Philosophy, 14, pp. 127-42.

Griffiths, Paul E. and Gray, Russell D. (1994) 'Developmental Systems and Evolutionary Explanation', Journal of Philosophy, 91(6), June, pp. 277-304.

Griffiths, Paul E. and Gray, Russell D. (1997) 'Replicator II - Judgment Day', Biology and Philosophy, 12, pp. 471-90.

Griffiths, Paul E. (2001) 'Genetic Information: A Metaphor in Search of a Theory', Philosophy of Science, 68(3), pp. 394-412.

Henrich, Joseph (2004) 'Cultural Group Selection, Coevolutionary Processes and Large-Scale Cooperation', Journal of Economic Behavior and Organization, 53(1), February, pp. 3-35.

Henrich, Joseph and Boyd, Robert (2001) 'Why People Punish Defectors: Why Conformist Transmission Can Stabilize Costly Enforcement of Norms in Cooperative Dilemmas', Journal of Theoretical Biology, 208(1), pp. 79-89.

Hodgson, Geoffrey M. (2005) 'Generalizing Darwinism to Social Evolution: Some Early Attempts', Journal of Economic Issues, 39(4), December 2005, pp. 899-914.

Hodgson, Geoffrey M. (2003) 'The Mystery of the Routine: The Darwinian Destiny of An Evolutionary Theory of Economic Change', Revue Économique, 54(2), Mars, pp. 355-84.

Hodgson, Geoffrey M. (2004) The Evolution of Institutional Economics: Agency, Structure and Darwinism in American Institutionalism (London and New York: Routledge).

Hodgson, Geoffrey M. and Knudsen, Thorbjørn (2004a) 'The Complex Evolution of a Simple Traffic Convention: The Functions and Implications of Habit', Journal of Economic Behavior and Organization, 54(1), pp. 19-47.

Hodgson, Geoffrey M. and Knudsen, Thorbjørn (2004b) 'The Firm as an Interactor: Firms as Vehicles for Habits and Routines', Journal of Evolutionary Economics, 14(3), July, pp. 281-307. 
Hodgson, Geoffrey M. and Knudsen, Thorbjørn (2006a) 'Why We Need a Generalized Darwinism: and Why a Generalized Darwinism is Not Enough', Journal of Economic Behavior and Organization, 61(1), September, pp. 1-19.

Hodgson, Geoffrey M. and Knudsen, Thorbjørn (2006b) 'Dismantling Lamarckism: Why Descriptions of Socio-Economic Evolution as Lamarckian are Misleading', Journal of Evolutionary Economics, 16(4), October, pp. 343-66.

Hodgson, Geoffrey M. and Knudsen, Thorbjørn (unpublished) 'Some Conjectures on the Growth of Complexity in Social and Economic Evolution', unpublished.

Hull, David L. (1988) Science as a Process: An Evolutionary Account of the Social and Conceptual Development of Science (Chicago: University of Chicago Press).

Jablonka, Eva and Szathmáry, Eörs (1995) 'The Evolution of Information Storage and Heredity', Trends in Ecology and Evolution, 10, pp. 206-11.

Joas, Hans (1993) Pragmatism and Social Theory (Chicago: University of Chicago Press).

Johnson-Laird, Philip N. (1981) 'Comprehension as the Construction of Mental Models', Philosophical Transactions of the Royal Society of London. Series B. Biological Sciences, 295, no. 1077, pp. 353-74.

Karmiloff, Kyra and Karmiloff-Smith, Annette (2001) Pathways to Language: From Fetus to Adolescent (Cambridge, MA: Harvard University Press).

Keller, Albert Galloway (1915) Societal Evolution: A Study of the Evolutionary Basis of the Science of Society (New York: Macmillan).

Keller, Laurent (ed.) (1999) Levels of Selection in Evolution: Monographs in Behavior and Ecology (Princeton: Princeton University Press).

Kubovy, Michael and Epstein, William (2001) 'Internalization: A Metaphor We Can Live Without', Behavioral and Brain Sciences, 24(4), pp. 618-25.

Knudsen, Thorbjørn (2002) 'The Significance of Tacit Knowledge in the Evolution of Human Language', Selection, 3(1), pp. 93-112.

Kurthen, Martin (2001) 'The Archeology of Internalism', Behavioral and Brain Sciences, 24(4), pp. 682-83.

Lee, Jeong Woong, Beebe, Kirk, Nangle, Leslie A., Jang, Jaeseon, Longo-Guess, Chantal M., Cook, Susan A., Davisson, Muriel T., Sundberg, John P., Schimmel, Paul and Ackerman, Susan L. (2006) 'Editing-defective tRNA Synthetase Causes Protein Misfolding and Neurodegeneration', Nature, 443, pp. 50-55. doi:10.1038/nature05096.

Luksha, Pavel O. (2003) 'Formal Definition of Self-reproductive Systems' Standish, Russell K., Bedau, Mark A. and Abbass, Hussein A. (eds) (2003) Artificial Life VIII: Proceedings of the Eighth International Conference on Artificial Life (Cambridge, MA: MIT Press), pp. 414-17.

Maynard Smith, John and Szathmáry, Eörs (1995) The Major Transitions in Evolution (Oxford: WH Freeman Press).

Maynard Smith, John (2000a), 'The Concept of Information in Biology', Philosophy of Science', Philosophy of Science, 67(2), pp. 177-194.

Maynard Smith, John (2000b), 'Reply to Commentaries', Philosophy of Science, 67(2), pp. 214-218. 
Mayr, Ernst (1974) 'Behavior Programs and Evolutionary Strategies', American Scientist, 62(6), pp. 650-659.

Mayr, Ernst (1988) Toward a New Philosophy of Biology: Observations of an Evolutionist (Cambridge, MA and London: Harvard University Press).

Michod, Richard E. (1999) Darwinian Dynamics: Evolutionary Transitions in Fitness and Individuality (Princeton: Princeton University Press).

Molofsky, Jane (1994) 'Population Dynamics and Pattern Formation in Theoretical Populations', Ecology, 1, pp. 30-39.

Nanay, Bence (2002) 'The Return of the Replicator: What is Philosophically Significant in a General Account of Replication and Selection', Biology and Philosophy, 17(1), January, pp. 109-21.

Nelson, Richard R. and Winter, Sidney G. (1982) An Evolutionary Theory of Economic Change (Cambridge, MA: Harvard University Press).

Neumann, John von (1966) Theory of Self-Reproducing Automata, ed. and completed by Arthur W. Burks (Urbana: University of Illinois Press).

Polanyi, Michael (1967) The Tacit Dimension (London: Routledge and Kegan Paul).

Prusiner, Stanley B. (1998) 'Prions', Proceedings of the National Academy of the Sciences, 95, November, pp. 13,363-13,383.

Putnam, Hilary (1995) Pragmatism (Oxford: Blackwell).

Reber, Arthur S. (1993) Implicit Learning and Tacit Knowledge: An Essay on the Cognitive Unconscious (Oxford and New York: Oxford University Press).

Ridley, Mark (2000) Mendel's Demon: Gene Justice and the Complexity of Life (London: Weidenfeld \& Nicolson).

Ritchie, David G. (1896) 'Social Evolution', International Journal of Ethics, 6(2), pp. 165-81.

Sarkar, Sahotra (2000) 'Information in Genetics and Developmental Biology: Comments on Maynard Smith', Philosophy of Science, 67(2), pp. 208-213.

Saunders, Peter T. and Ho, Mae-Wan (1976) 'On the Increase in Complexity in Evolution', Journal of Theoretical Biology, 63, pp. 375-84.

Saunders, Peter T. and Ho, Mae-Wan (1981) 'On the Increase in Complexity in Evolution II: The Relativity of Complexity and the Principle of Minimum Increase', Journal of Theoretical Biology, 90, pp. 515-30.

Shannon, Claude E., and Warren Weaver (1949) The Mathematical Theory of Communication (Chicago: University of Illinois Press).

Simon, Herbert A. (1990) 'A Mechanism for Social Selection and Successful Altruism', Science, 250, 21 December, pp. 1665-8.

Sipper, Moshe (1998) 'Fifty Years of Research on Self-Replication: An Overview', Artificial Life, 4(3), pp. 237-57.

Sober, Elliott and Wilson, David Sloan (1998) Unto Others: The Evolution and Psychology of Unselfish Behavior (Cambridge, MA: Harvard University Press). 
Sperber, Dan (2000) 'An Objection to the Memetic Approach to Culture', in Aunger, Robert (ed.) (2000) Darwinizing Culture: The Status of Memetics as a Science (Oxford and New York: Oxford University Press), pp. 162-73.

Stadler, Bärbel M. R., Stadler, Peter F., and Wagner, Günter P. (2001) 'The Topology of the Possible: Formal Spaces Underlying Patterns of Evolutionary Change', Journal of Theoretical Biology, 213, pp. 241-274.

Sterelny, Kim, Smith, Kelly C. and Dickison, Michael (1996) 'The Extended Replicator', Biology and Philosophy, 11, pp. 377-403.

Sterelny, Kim (2000) 'The "Genetic Program" Program: A Commentary on Maynard Smith on Information in Biology', Philosophy of Science, 67(2), pp. 195-201.

Szathmáry, Eörs (2000) 'The Evolution of Replicators', Philosophical Transactions: Biological Sciences, 355, no. 1403, November 29, pp. 1669-1676.

Tomasello, Michael (1999) The Cultural Origins of Human Cognition (Cambridge, MA: Harvard University Press).

Veblen, Thorstein B. (1899) The Theory of the Leisure Class: An Economic Study in the Evolution of Institutions (New York: Macmillan).

Wimsatt, William C. (1999) 'Genes, Memes, and Cultural Heredity', Biology and Philosophy, 14(2), April, pp. 279-310.

Wolfram, Stephen (1984) 'Universality and Complexity in Cellular Automata' Physica, 10D, pp. 1-35.

Wolfram, Stephen (2002) A New Kind of Science (Champaign, IL: Wolfram Media, Inc.). 